\title{
Bilateral pallidotomy for treatment of Parkinson's disease induced corticobulbar syndrome and psychic akinesia avoidable by globus pallidus lesion combined with contralateral stimulation
}

\author{
M Merello, S Starkstein, M I Nouzeilles, G Kuzis, R Leiguarda
}

\begin{abstract}
Objective-Posteroventral pallidotomy (PVP) has proved to be an effective method for the treatment of Parkinson's disease. However, data on bilateral procedures are still limited. To assess the effects of bilateral globus pallidus (GPi) lesion and to compare it with a combination of unilateral GPi lesion plus contralateral GPi stimulation (PVP+PVS), an open blind randomised trial was designed.

Methods-A prospective series of patients with severe Parkinson's disease refractory to medical treatment, and severe drug induced dyskinesias, were randomised either to simultaneous bilateral PVP or simultaneous PVP+PVS. All patients were assessed with the core assessment programme for intracerebral transplantation (CAPIT), and a comprehensive neuropsychological and neuropsychiatric battery both before surgery and 3 months later.

Results-The severe adverse effects found in the first three patients subjected to bilateral PVP led to discontinuation of the protocol. All three patients developed depression and apathy. Speech, salivation, and swallowing, as well as freezing, walking, and falling, dramatically worsened. By contrast, all three patients undergoing PVP+PVS had a significant motor improvement.

Conclusion-Bilateral simultaneous lesions within the GPi may produce severe motor and psychiatric complications. On the other hand, a combination of PVP+ PVS significantly improves parkinsonian symptoms not associated with the side effects elicited by bilateral lesions. (F Neurol Neurosurg Psychiatry 2001;71:611-614)
\end{abstract}

Keywords: bilateral pallidotomy; deep brain stimulation; globus pallidus stimulation; psychic akinesia

Unilateral lesion of the posteroventral portion (PVP) of the internal globus pallidus (GPi) is an effective method for the treatment of Parkinson's disease. ${ }^{1-5}$ However, data on bilateral procedures are scant and mainly based on non-controlled studies. ${ }^{6-12}$

Unilateral posteroventral GPi stimulation (PVS) may be as effective as unilateral PVP, with a similar frequency of side effects. ${ }^{13}$ Contemporaneous bilateral PVS has also been reported to be a safe and efficient technique, ${ }^{14}{ }^{15}$ but results of a combination of both PVP and PVS have only been described in a single study. ${ }^{16}$

Iacono et al carried out simultaneous bilateral pallidotomy on 68 patients. The low frequency of side effects led the authors to conclude that there is no reason for staged lesions, thus reducing the risk of two operations, lowering the cost of the procedures, and improving preoperative target planning of the second side. ${ }^{6}$

Based on the above findings, we designed an open blind prospective randomised trial initially developed to randomise either to bilateral PVP or unilateral PVP plus contralateral PVS, with a minimum of six patients in each group.

\section{Patients and methods}

After ethics committee approval and signed informed consent, a prospective series of patients with PD was included in the protocol. All patients met the idiopathic Parkinson's disease (IPD) criteria of the brain bank of London, ${ }^{17}$ including invalidating bilateral and symmetric Parkinson's disease symptoms refractory to medical treatment (severe motor fluctuations with unacceptable quality of life either in the on or off state despite the use of levodopa, dopamine agonists, COMT inhibitors, or apomorphine, among others) and severe drug induced dyskinesias. None of the patients were demented, or had severe medical conditions or focal lesions on MRI.

\section{NEUROLOGICAL EXAMINATION}

Neurological examination was performed according to the core assessment programme for intracerebral transplantation (CAPIT) described elsewhere. ${ }^{18}$ Briefly, it consists of serial evaluations in off and on states during a fixed preoperative and postoperative period. Evaluation comprises a battery of scales and timed tests such as UPDRS, activities of daily living (ADL), walking time, and tapping test together with a dyskinesia score. Baseline scores were calculated by averaging the last two evaluations within 1 week before surgery, and postoperative scores were analysed at 3 month follow up.

PSYCHIATRIC AND NEUROPSYCHOLOGICAL EVALUATION

Each patient was assessed by a neuropsychologist and a psychiatrist blind to the purpose of the study 1 month before and between 2 to 3 months after surgery, using the following 
Table 1 Mean (SD) preoperative and postoperative off stage UPDRS scores for bilateral posteroventral pallidotomy (BIL-PVP) versus posteroventral pallidotomy plus posteroventral stimulation $(P V P+P V S)$

\begin{tabular}{llllll}
\hline & \multicolumn{2}{l}{$B I L-V P$} & & \multicolumn{2}{l}{$P V P+P V S$} \\
\cline { 2 - 3 } \cline { 5 - 6 } & Pre & Post & & Pre & Post \\
\hline UPDRS & $26.3(4.1)$ & $31(12.1)$ & & $28.5(11.5)$ & $18.5(4.5)$ \\
Bradykinesia & $7.33(2)$ & $6.33(4.1)$ & & $7.6(6)$ & $4.66(1.5)$ \\
Rigidity & $2.66(2.3)$ & $2.5(0.57)$ & & $2.66(2.3)$ & $1.66(1.52)$ \\
Rest tremor & $1(1)$ & $0.33(0.5)$ & & $2.5(0.7)$ & $0(0)$ \\
PIGD $^{\star}$ & $9(5.1)$ & $11.3(1.7)$ & & $7(2.6)$ & $4(3.7)$ \\
\hline
\end{tabular}

${ }^{\star} F(1,4)=5.1 ; \mathrm{p}<0.05$. Hand bradykinesia (UPDRS items $23+24+25$ ), rigidity (item 22 ) and resting tremor (item 20) scores were not statistically different. A composite subscore of postural instability and gait disorders (PIGD) (UPDRS items 28+29+30) showed significant impairment after BIL-PVP. Dyskinesia scores significantly improved in trunk, head, and bilaterally in upper and lower limbs after either procedure.

instruments: Hamilton depression score; functional independence measurement; apathy, irritability, and mania scales; Raven's progressive matrices; Wisconsin card sorting test (WCST); controlled oral word association test; Buschke selective reminding test; Benton visual retention test; digit span; Perdue pegboard; Hooper visual organisation test; Benton visual form discrimination; and block design (WAIS).

SURGICAL PROCEDURE

Patients were randomised to either procedure the day before surgery. The surgical procedure has been described elsewhere ${ }^{4}{ }^{13}$; briefly, it was microelectrode guided and both sides were operated on in a single intervention. At the site determined by electrophysiological parameters, a 75 degree 60 second lesion was performed either unilaterally or bilaterally. A 3387 DBS electrode placed in the motor part of the GPi was connected to an internal pulse generator (Itrel II Medtronic, Minneapolis, USA) in those patients who received a stimulator contralateral to the lesion. Three months after surgery three dimensional MRI was carried out to confirm lesion placement and volume or lead positioning.

STATISTICAL ANALYSIS

Statistical analyses were carried out using two way analysis of variance (ANOVA) with repeated measures and post hoc paired $t$ test comparisons. Frequency distributions were calculated by $\chi^{2}$ and cells $<5$ were Yates corrected. All $\mathrm{p}$ values were two tailed and the threshold for significance was set at $\mathrm{p}<0.05$. Values are expressed as mean (SD).

\section{Results}

The severe adverse effects seen in the first three patients subjected to bilateral PVP led to discontinuation of the protocol.

DEMOGRAPHIC FINDINGS

There were five men and one woman. For BIL-PVP and PVP+PVS respectively, mean ages were 67 (6.4) and 55 (9.5) (NS), mean disease duration was 10.3 (5) and 10 (4.9) (NS), and mean duration of levodopa treatment was 10.3 (4) and 9.3 (4.9) (NS). There were no differences in side of initial symptom or more affected side between either group. The mean total motor section scores from the unified Parkinson's disease rating scale version 3.0 (UPDRS) were 26.3 (4.1) and 28.5 (11.5) (NS) for BIL-PVP and PVP+PVS, respectively.

All six patients were Hoehn and Yahr stage IV.

NEUROLOGICAL FINDINGS

UPDRS parts I and II, which evaluate mentation, behaviour, mood, and ADL, significantly deteriorated after bilateral PVP, but improved after the combined technique. Mean total preoperative and postoperative values for BILPVP and PVP+PVS were 24.6 (5.5) v 30.3 (11.0) and $33.3(6.6 \vee 19$ (9.)1, respectively $(F(1,4)=7.03 ; \mathrm{p}<0.05)$; speech, salivation and swallowing explained most of the changes $(\mathrm{p}<0.01)$.

The UPDRS part III subscore that evaluates motor performance significantly improved after the combined technique $(F(1,4)=4.17$; $\mathrm{p}<0.05)$, whereas no difference was found in bilaterally lesioned patients. Mean values for UPDRS motor score for preoperative and postoperative BIL-PVP and PVP+PVS were $26.3(4.1)$ v 31 (12.1) and $28.5(11.5)$ v 18 (4.5), respectively.

A composite subscore of hand bradykinesia (UPDRS items $23+24+25$ ) was analysed. Values for preoperative and postoperative BILPVP and PVP+PVS were not statistically different $(F(1,4)=0.86)$. Neither were the values for rigidity (UPDRS item 22) $(F(1,4)=0.25)$ and resting tremor (UPDRS item 20$)(F(1,3) 3.82)$. A composite subscore of postural instability and gait disorders (UPDRS items 28+29+30) (PIGD) showed significant impairment after BIL-PVP $(F(1,4)=5.1 ; \mathrm{p}<0.05)$. Dyskinesia score significantly improved in the trunk and head, and bilaterally in the upper and lower limbs after either procedure $(F(5,20)=3.86 ; \mathrm{p}<0.01$, table $1)$.

PSYCHIATRIC EVALUATION

There was a significant surgery effect on neuropsychiatric evaluation $(F(4,16) \quad 6.39$ $\mathrm{p}<0.002$ ), with a significant deterioration on Hamilton depression score $(p<0.004)$ and apathy score $(p<0.0001)$ after BIL-PVP.

\section{NEUROPSYCHOLOGICAL EVALUATION}

Comprehensive postoperative neuropsychological evaluation was not possible in the three bilaterally lesioned patients, so results were not computed.

\section{SIDE EFFECTS}

Postoperatively, patients with bilateral lesion had unintelligible speech, marked drooling, and swallowing difficulties, initially requiring feeding through nasogastric intubation, and later on feeding with soft food. Freezing gait deteriorated without beneficial effect of increasing levodopadopa doses, requiring daily physical therapy to maintain mobility. Patients presented apathy and a severe loss of initiative, motivation, and motor drive. All those symptoms persisted at the 3 month postoperative evaluation. 
SURGICAL RESULTS

Brain MRI 3 months after surgery showed that all nine lesions and the three electrodes were located entirely within the GPi. Coordinates of the lesion/lead as well as lesion volumes were not significantly different between the groups.

\section{Discussion}

On the basis of a few non-controlled studies on bilateral pallidotomy either with or without beneficial effect or complications, it has been posited that the outcome is critically influenced by a poorly or accurately placed lesion rather than by the effect of placing simultaneous bilateral pallidal lesions. ${ }^{12}$

Schuurman et $a l^{11}$ have described three patients undergoing bilateral pallidotomy performed at an interval of over 6 weeks in whom neither neuropsychiatric complications nor speech, swallowing or salivation difficulties, or PIGD deterioration were recorded. In this series, facial paresis and optic tract damage were instead reported, but we think that they corresponded to lesion extension to the internal capsule and optic tract. In agreement, Iacono $e t a l^{6}$ reported 68 bilateral procedures, out of which 48 were contemporaneous. They reported no significant increase in the risk of complications for patients undergoing contemporaneous pallidotomy compared with staged procedures.

By contrast, Ghika et $a l^{19}$ documented that severe neuropsychological and psychiatric changes could result from these procedures and in support, Roberts et $a l^{20}$ published an unacceptable risk of swallowing or speech disorders even in staged procedures. In the latest and most comprehensive review on bilateral pallidotomy, Ghika et al described four patients who underwent simultaneous bilateral pallidotomy complicated with loss of psychic self activation, with emotional and cognitive deficits, as well as disabling corticobulbar dysfunction and apraxia of eyelid opening, which make surgery undesirable. ${ }^{14}$

Before further speculation, we should point out the limited number of patients included in our series due to an unacceptable rate of complications that made us halt the protocol before reaching the initial planned number of cases, as well as the short term follow up. Whether reported complications can resolve in the medium term or at long term evaluation should be tested on future follow up.

Our present findings argue against the possibility that lesion inaccuracy is responsible for the unacceptable rate of side effects of bilateral procedures $^{6}{ }^{12}$ as targets were confirmed by microrecording, lesions checked by MRI and the same criteria were followed either for lesioned or stimulated patients. Besides, no visual field or pyramidal defects resulting from optic tract or internal capsule damage were manifested, so we must assume that factors other than lesion placement should be responsible for such a high and unacceptable incidence of complications.

In addition to corticobulbar symptoms, ${ }^{21-23}$ ischaemic, hypoxic, or haemorrhagic bilateral pallidal lesions may cause profound behavioural and personality changes. One of the main findings of the present study was the significant increment in depression and apathy scores after simultaneous bilateral PVP. Starkstein et al reported a significant association between apathy and unilateral lesions to the medial portion of the globus pallidus. ${ }^{24}$ Bilateral ischaemic lesions to the globus pallidus were reported to produce a severe loss of drive and motivation known as "psychic akinesia". The ansa lenticularis, one of the main outputs of the globus pallidus, goes through the posterior limits of the internal capsule and ends in the pedunculopontine nucleus, which sends monosynaptic projections to the motor neurons in the anterior horn. Starkstein et al speculated that the association between depression and apathy after ventral striatal lesions may result from disruption of biogenic amine pathways and damage to the contiguous internal capsule. ${ }^{25}$

Although the precise mechanism of stimulation is still unknown, the similarity of effects induced both by the lesion and high frequency electrical stimulation leads us to consider the overall effect of the second as a neuroinhibition. Such inhibition would result either from direct neuronal blockade, stimulation of inhibitory structures, or no physiological high frequency synchronous activation of a very large population of neurons or fibres that deliver a disrupted message incomprehensible for the projection targets. ${ }^{26}{ }^{27}$ Therefore, it remains to be elucidated why bilateral ablative procedures are liable to induce such severe side effects that could be avoided by replacing one of the lesions by a stimulator placed according to the same criteria and localisation as the lesion.

In conclusion, despite the lack of a clear explanation for the differences in the outcome between both techniques, findings of the present study strongly support the combination of PVP+PVS. Whether or not PVP+PVS produced a comparable effect to bilateral PVS presents important economic implications and should be tested in future studies to define the procedure of choice for bilateral pallidal surgery.

\footnotetext{
1 Dogali M, Fazzini E, Kolodny E, et al. Stereotactic ventral pallidotomy for Parkinson's disease. Neurology 1995;45:753-61.

2 Baron MS, Vitek JL, Bakay RAE, et al. Treatment of advanced Parkinson's disease by internal globus pallidotomy: 1 year results of a pilot study. Ann Neurol pallidotomy: 1 year

3 Lang A, Lozano A, Montgomery E, et al. Effect of GPi pallidotomy on motor function in Parkinson's disease. Lancet 1995;346:1383-7

4 Merello M, Nouzeilles MI, Cammarota A, et al. Comparison of 1 year follow up evaluation of patients with indication for pallidotomy who did not undergo surgery versus patients with Parkinson's disease who did undergo pallidotomy: a case control study. Neurosurgery 1999;44: $461-7$.

5 de Bie RMA, de Haan RJ, Nijssen PCG, et al. Unilateral pallidotomy in Parkinson's disease: a randomised, singleblind, multicentre trial. Lancet 1999;354:1665-9.

6 Iacono RP, Shima F, Lonser RR, et al. The results, indications, and physiology of posteroventral pallidotomy for patients with Parkinson's disease. Neurosurgery 1995;36: $1118-27$.

7 Iacono R, Lonser R. Reversal of Parkinson's akinesia by pallidotomy. Lancet 1994;343:1095-6.
} 
8 Svennilson E, Torvik A, Lowe R, et al. Treatment of parkinsonism by stereotactic thermo lesions in the pallidal region. A clinical evaluation of 81 cases. Acta Psychiatr Neurol

9 Iacono RP, Lonser RR, Mandybur G, et al. Stimulation of the globus pallidus in Parkinson's disease. Br $\mathcal{F}$ Neurosurg 1995;9:505-10.

10 Laitinen IV. Leksell's posteroventral pallidotomy in the treatment of Parkinson's disease. F Neurosurg 1992;76:5361.

11 Schuurman R, de Bie R, Speelman J, et al. Bilateral pallidotomy in advanced Parkinson's disease in three patients. Mov Disord 1997;12:752-5.

12 Bronstein J, De Salles A, De Long M. Stereotactic pallidotomy in the treatment of Parkinson disease. Arch Neurol 1999,56:1064-9.

13 Merello M, Nouzeilles MI, Kuzis G, et al. Unilateral radiofrequency lesion versus electrostimulation of posteroventral pallidum: a prospective randomised comparison. Mov Dispallidum: a prospecti $1999 ; 14: 50-6$.

14 Ghika J, Ghika-Schmid F, Frankhauser H, et al. Bilateral contemporaneous posteroventral pallidotomy for the treatment of Parkinson disease: neuropsychological and neurological side effects. 7 Neurosurg 1999,91:313-21.

15 Siegfried J, Lippitz B. Bilateral chronic electrostimulation of ventroposterolateral pallidum: a new therapeutic approach for alleviating all parkinsonian symptoms. I Neurosurg 1994;35:1126-30

16 Galvez-Jimenez N, Lozano A, Tasker R, et al. Pallidal stimulation in Parkinson's disease patients with a prior unilateral pallidotomy. Can $\mathcal{F}$ Neurol Sci 1998;4:300-5.

17 Hughes AJ, Daniel SE, Kilford L, et al. Accuracy of clinical diagnosis of idiopathic Parkinson's disease: a clinicopathological study of 100 cases. 7 Neurol Neurosurg Psychiatry 1992;55:181-4.
18 Langston J, Widner I, Goetz C, et al. Core assessment program for intracerebral transplantation (CAPIT). Mov Dis92:7:2-13.

19 Ghika J, Favre J, Frankhauser H, et al. Neurological and neuropsychological complications of bilateral contemporaneous pallidotomy in Parkinson disease Neurology 1996;46: A417.

20 Roberts J, Helbrun M. Staged bilateral ventroposterolateral pallidotomy for Parkinson's disease. Neurology 1997;48: S53.004

21 Laitinen LV. Brain targets in surgery for Parkinson's disease. Results of a survey of neurosurgeons. F Neurosurg 1985;62: 349-51.

22 Scott R, Gregory R, Hines N, et al. Neuropsychological, neurological and functional outcome following pallidotomy for Parkinson's disease. A consecutive series of eight simul(19) 121:659-75.

23 Sutton JP, Couldwell W, Lew MF, et al. Ventroposterior medial pallidotomy in patients with advanced Parkinson's disease. Neurosurgery 1995;36:1112-17.

24 Starkstein SE, Berthier ML, Leiguarda R. Psychic akinesia following bilateral pallidal lesions. Int $f$ Psychiatry Med

25 Starkstein SE, Fedoroff JP, Price TR, et al. Apathy following cerebrovascular lesions. Stroke 1993;24:1625-30.

26 Benabid AL, Pollak P, Hoffmann D, et al. Chronic high-frequency thalamic stimulation in Parkinson's disease. In: Koller WC, Paulson G, eds. Therapy of Parkinson's disease. 2nd ed. New York: Marcel Dekker, 1995:381-402.

27 Benazzouz A, Piallat B, Pollak P, et al. Responses of substantia nigra pars reticulata and globus pallidus complex to high frequency stimulation of the subthalamic nucleus in rats: electrophysiological data. Neurosci Lett 1995;189:7780

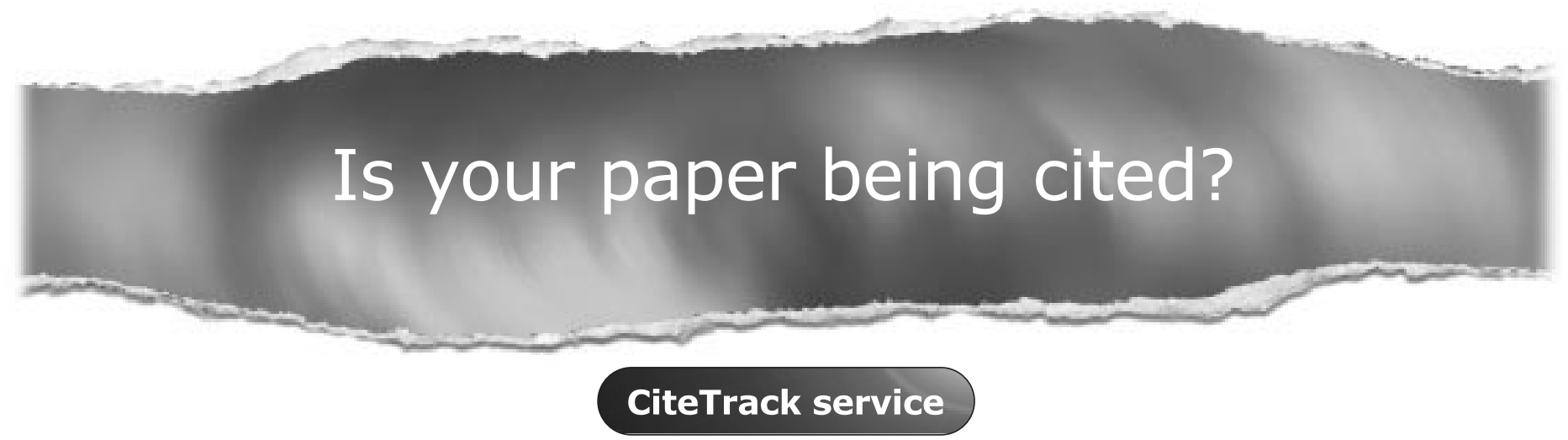

CiteTrack will alert you by email whenever new content in the Journal of Neurology, Neurosurgery, and Psychiatry or a participating journal is published that matches criteria you want to track Topics: Tell CiteTrack which words or subjects to watch for in new content Authors: Be alerted whenever key authors you are following publish a new paper Articles: Know whenever a paper of interest to you is referenced by another paper

\section{www.jnnp.com}

